\title{
Capital cultural y rendimiento académico de estudiantes normalistas en Sonora, México*
}

\section{Jesús Tánori Quintana}

https://orcid.org/00oo-0002-6485-2267 Instituto Tecnológico de Sonora, México jesus.tanori@itson.edu.mx

\section{José Ángel Vera Noriega}

https://orcid.org/oooo-0003-2764-4431 Universidad de Sonora, México jose.vera@unison.mx

\section{Adolfo Álvarez Quintero}

https://orcid.org/oooo-0001-7088-5199 Instituto Tecnológico de Sonora, México adolfo.alvarez11123@potros.itson.edu.mx

\section{Francisco Fernando Durazo Salas}

https://orcid.org/oooo-ooo1-7229-6953 TECNOESTATA SC, México durazof@gmail.com

\section{Resumen}

En los últimos años evaluaciones internacionales y nacionales han presentado resultados poco favorables para el sistema educativo en México, centrando el análisis en la calidad de los profesores. Esta problemática ha sido similar en el concurso de oposición para el ingreso a la profesión docente y pone en duda la eficiencia de la formación inicial a cargo de las escuelas normales mexicanas. El objetivo del presente artículo es estudiar la relación entre origen social y rendimiento académico de una comunidad estudiantil de tres planteles de la Escuela Normal en Sonora. El estudio analiza una cohorte de 268 estudiantes mediante el uso operativo de los cinco tipos de capital cultural que, como variables, contribuyen al análisis del rendimiento académico. Además, se determinó el origen social de los estudiantes con la prueba de conglomerados de K-medias. Los resultados muestran, a través de un análisis del coe-

* Este artículo de investigación formó parte del proyecto "Trayectorias escolares en estudiantes normalistas en el Estado de Sonora". El proyecto estuvo financiado por la Universidad de Sonora y el Instituto de Formación Docente del Estado de Sonora (IFODES). Artículo proveniente de tesis de maestría de Adolfo Álvarez Ouintero en el periodo 2012-2015, bajo el nombre de "Origen social y rendimiento académico de una cohorte de estudiantes de la Escuela Normal en Sonora" para obtener el grado de Maestro en Innovación Educativa, bajo la tutela del doctor José Ángel Vera Noriega de la Universidad de Sonora.

Recepción: 10/07/2020 | Envío a pares: 16/11/2020 | Aceptación por pares: 07/01/2021 | Aprobación: 09/02/2021 
ISSN 0123-1294 | e-ISSN 2027-5358 | Educ.Educ. Vol. 24. No. 1 | Febrero-abril de 2021 | pp. 53-70

Universidad de La Sabana | Facultad de Educación

ficiente de correlación de Pearson, que el capital familiar, escolar y económico del estudiante se relaciona con el rendimiento académico. Después se realizó un análisis de regresión lineal donde el valor más alto de beta fue para la variable capital escolar. Se concluye, en un marco de desigualdad social, que el capital escolar y el económico son los factores que intervienen en el logro educativo de los estudiantes que se están formando como formadores.

\section{Palabras clave (Fuente: tesauro de la Unesco)}

Capital social; enseñanza superior; evaluación de la educación; formación de docentes; rendimiento escolar. 


\title{
Cultural Capital and Academic Performance of Normal School Students in Sonora, Mexico*
}

\begin{abstract}
In recent years, international and national test results have been unfavorable for Mexico's educational system, turning the spotlight on teachers' quality. This problem has also occurred in the public teaching entrance examination and calls into question the efficiency of the initial training provided by Mexican normal schools. This paper intends to study the relationship between a student community's social origin and academic performance in three normal school sites in Sonora. It analyzes a cohort of 268 students through the five cultural capital types that, as variables, contribute to exploring academic performance. Besides, it determines the social origin of the students through K-means clustering. Through an analysis of the Pearson correlation coefficient, the results show that the student's family, school, and economic capitals are associated with academic performance. Afterward, a linear regression analysis was carried out where the highest Beta value was for the school capital variable. In conclusion, in a framework of social inequality, the school and economic capitals take part in the educational achievement of students who are being trained as teachers.
\end{abstract}

\section{Keywords (Source: Unesco Thesaurus)}

Social capital; teacher education; educational evaluation; teacher training; academic achievement.

* This research article was part of the project "Trayectorias escolares en estudiantes normalistas en el Estado de Sonora," funded by the Universidad de Sonora and the Instituto de Formación Docente del Estado de Sonora (ifodes). It derives from Adolfo Álvarez Quintero's thesis in 2012-2015 titled "Origen social y rendimiento académico de una cohorte de estudiantes de la Escuela Normal en Sonora" to obtain his Master's Degree in Educational Innovation, supervised by José Ángel Vera Noriega, PhD, Universidad de Sonora. 


\section{Capital cultural e desempenho acadêmico de estudantes normalistas em Sonora, México*}

\section{Resumo}

Nos últimos anos, avaliações nacionais e internacionais vêm apresentando resultados pouco favoráveis para o sistema educacional no México, centralizando a análise na qualidade dos professores. Essa problemática vem sendo similar no concurso de oposição para ingressar na profissão docente e coloca em dúvida a eficiência da formação inicial sob a responsabilidade das escolas normais mexicanas. O objetivo deste trabalho é estudar a relação entre origem social e desempenho acadêmico de uma comunidade estudantil de três turmas da Escola Normal em Sonora. Neste estudo, analisa-se uma coorte de 268 estudantes mediante o uso operacional dos cinco tipos de capital cultural que, como variáveis, contribuem para analisar o desempenho acadêmico. Além disso, foi determinada a origem social dos estudantes com a análise de conglomerados de K-medias. Os resultados mostram, por meio de uma análise do coeficiente de correlação de Pearson, que o capital familiar, escolar e econômico do estudante se relaciona com o desempenho acadêmico. Em seguida, foi realizada uma análise de regressão linear em que o valor mais alto de beta foi para a variável capital escolar. Conclui-se, no âmbito de desigualdade social, que o capital escolar e o econômico são os fatores que intervêm na conquista educacional dos estudantes que estão sendo formados como formadores.

\section{Palavbras-chave (Fonte: tesauro da Unesco)}

Capital social; ensino superior; avaliação educacional; treinamento de professor; rendimento escolar.

\footnotetext{
Este artigo de pesquisa fez parte do projeto "Trajetórias escolares em estudantes normalistas no Estado de Sonora". O projeto foi financiado pela Universidad de Sonora e pelo Instituto de Formación Docente del Estado de Sonora. Artigo proveniente da dissertação em Inovação Educacional de Adolfo Álvarez Quintero entre 2012 e 2015, sob o título "Origen social y rendimiento académico de una cohorte de estudiantes de la Escuela Normal en Sonora" ("Origem social e desempenho acadêmico de um coorte de estudantes da Escola Normal em Sonora"), sob a tutela do doutor José Ángel Vera Noriega da Universidad de Sonora.
} 
Actualmente la búsqueda de la calidad educativa y el manejo de un paradigma sostenido por la competitividad y la eficiencia han provocado que se cuestionen tanto la formación inicial como el desarrollo profesional, no solo de los estudiantes sino también de los profesores. Valoraciones del desempeño, como el Programa para la Evaluación Internacional de los Estudiantes (PISA - Programme for International Student Assessment), las del Centro Nacional de Evaluación para la Educación Superior (CENEVAL) y el Examen Nacional de Conocimientos, Habilidades y Competencias Docentes (ENCHCD), COnocido también como examen de oposición, han generado una controversia puntual, al poner en tela de juicio la efectividad del sistema educativo y la idoneidad de los docentes mexicanos, a pesar de estar dirigidas a actores y niveles educativos distintos. Controversia con la cual los profesores en servicio docente tendrán que lidiar, así como quienes se encuentran en plena formación cuando egresen de sus estudios superiores (INEE, 2017; Santiago et al., 2012).

Dichas evaluaciones han mostrado resultados poco favorables, aun cuando se ha emprendido una serie de acciones, programas y reformas por parte del gobierno mexicano, como el Programa de Fortalecimiento Académico de los Estudiantes de las Escuelas Normales (PFAEEN), generado a partir de los resultados del examen de ingreso a la profesión docente y enfocado en los estudiantes con un rendimiento bajo. No obstante, en ninguno de los casos se ha buscado conocer la opinión de uno de los actores principalmente afectados: los profesores, ni de los que se encuentran en servicio docente ni de los que están en formación inicial en las distintas escuelas normales y universidades pedagógicas nacionales (DGESPE, 2013; Medrano, Ángeles y Morales, 2017).

Por su parte, Sandoval (2013) ha dejado ver la falta de información sobre los docentes de educación básica, la cual sigue constituyendo un vacío en el conocimiento del sector, compartiendo así el señalamiento hecho por Ibarrola (2014) en referencia a la contradicción entre el papel protagónico que se le otorga al maestro de educación primaria y el desconocimiento de las actividades que desempeña. Si bien en México los estudios sobre el inicio de la docencia en la educación básica constituyen una línea de investigación reciente y poco explorada, son considerados fundamentales para el fortalecimiento de la función docente y el diseño de propuestas que apoyen su mejor desarrollo y formación (Sandoval y Villegas, 2013). Por ello, resulta relevante llevar a cabo estudios que ayuden a documentar quiénes son los profesores en México. Tal como lo menciona Arnaut (2004, p. 37), "el magisterio ya no es lo que fue o, mejor dicho, ya no solo es lo que había sido".

\section{Revisión de la literatura}

Distintas investigaciones han indicado que las trayectorias escolares exitosas en educación superior están relacionadas con el promedio obtenido por el alumno en la escolarización inmediata anterior (preparatoria o bachillerato), con la percepción de apoyo e interés que perciba de los padres, con los hábitos y condiciones de estudio y, sobre todo, con los estilos que ejerza para lograr los aprendizajes de manera independiente (Barahona, 2014; Cárcamo y Rodríguez; 2015; Da Cuña et al., 2014; Garbanzo, 2013; González, 2013; Simón et al., 2017).

En este contexto, los estudios han generado evidencia suficiente para suponer que el capital familiar relacionado con la escuela se asocia con la percepción de los padres o tutores sobre la importancia del conocimiento y su transmisión para promover no solo la dinámica social, sino la equidad y la justicia. Este capital familiar en educación se construye de variadas formas, de acuerdo con el interés exhibido y dentro de un circuito en el cual la educación es vista como algo importante que, a su vez, conlleva la evaluación de la moratoria laboral de los estudiantes y condiciona la comprensión y aprecio que tengan los padres acerca de la educación, como un bien o patrimonio que se hereda (Arango, Quinteroy Mendoza, 2004; Enciso, 2013; Marqués y Gil, 2015). 
Por otro lado, el capital educativo se refiere al conjunto de habilidades y competencias que el alumno ha desarrollado a partir de la inversión educativa y que lo coloca en un plano de autopercepción en donde se hace posible llevar a cabo una discusión sobre la importancia, deseabilidad y necesidad de continuar invirtiendo en los procesos de educación. Este capital escolar incluye los promedios, el historial de aprobación y reprobación, las preferencias por algunas materias y las vocaciones por diferentes competencias profesionales, factores que se asocian con la permanencia escolar (Fonseca, 2018).

Tanto el capital familiar como el educativo, cuando se integran, generan una amalgama que nos indica el capital cultural, que está compuesto por la mecánica y los procesos de percepción subjetiva, asociada, por una parte, a los tutores - que pueden ver en la moratoria laboral una ventaja para el desarrollo del alumno y de la familia-y, por otra, al alumno, quien percibe, a través de procesos metacognitivos, que se encuentra en una condición de privilegio para desarrollar habilidades y competencias profesionales. A este capital cultural se le añade el capital económico, ya que la literatura muestra que la condición socioeconómica es fundamental para el desarrollo de habilidades académicas, ya que estas requieren de excedentes de capital monetario y excedentes de tiempo (Chaparro, González y Caso, 2016).

En la conjunción de capital educativo, económico y familiar se encuentra una zona en la cual las condiciones económicas permiten promover la demora laboral y motivar el desarrollo de competencias profesionales. Surge así una condición en la que el alumno genera una trayectoria exitosa con el apoyo de los padres, tanto de tipo económico como afectivo-emocional. Otra trayectoria tiene que ver con aquellos desposeídos que requieren del establecimiento de oportunidades a través del recurso fiscal para lograr desarrollar una meta asociada al término de una licenciatura con éxito. Se indaga en las variables relacionadas con cada uno de los tipos de capital para conformar un modelo y establecer perfiles relacionados con trayectorias sociales y estar en condiciones de observar cuál de estos factores conectados con los diferentes capitales es el que ocupa el mayor poder explicativo en relación con el logro académico (Bernardi y Cebolla, 2014; Bracho, 1990; Flores, Stadhagen y Reyes, 2014).

Por otro lado, la necesidad de documentar el perfil que deben presentar los futuros docentes para el desempeño de su carrera profesional debe ser estudiada en dos momentos: el primero de ellos, en el cual se enfoca esta investigación, es el inicio del análisis y la observación de perfiles de los estudiantes normalistas durante su formación inicial, estableciendo las bases para el segundo momento, donde se estudian los desempeños del profesor dentro del aula. En ambos casos, se busca contribuir al conocimiento de las variables psicológicas, vocacionales, sociales, culturales y educativas que permitan identificar un maestro exitoso desde su ingreso, durante la formación de su carrera profesional y al egreso de la formación inicial. Para lograr lo anterior se llevan a cabo mediciones con variables digitales que permiten un análisis explicativo del logro académico a partir de los diferentes capitales en segundo y cuarto semestre lectivos de formación del docente en la escuela normal.

\section{Objetivo y método}

El objetivo es determinar la relación entre el origen social de los normalistas, medido a través de un indicador de capital cultural, y el logro académico de una cohorte de cuatro semestres de la licenciatura en educación primaria de tres planteles de la Escuela Normal en Sonora. En cuanto al enfoque metodológico, la investigación es de tipo explicativo y se basa en un estudio censal de una cohorte de estudiantes del programa en Licenciatura en Educación Básica analizada durante los dos primeros años de su formación como docentes. El estudio trabajó con registros institucionales proporcionados por servicios estudiantiles de las tres escuelas normales participantes en el análisis, con información proporcionada por el IFODES 
y mediante la aplicación del instrumento de Evaluación y Valores Socioculturales de Vera y Aragón (2008) a los estudiantes de la cohorte seleccionada.

\section{Muestra y muestreo}

La investigación se realizó con la cohorte 201115 de estudiantes de la Licenciatura en Educación Primaria (LEP), pertenecientes a las tres escuelas normales del estado de Sonora que ofertan dicho programa: la Escuela Normal del Estado de Sonora (ENES), ubicada en la ciudad de Hermosillo; la Escuela Normal Rural Gral. Plutarco Elías Calles (ENR El Quinto), ubicada en el municipio de Etchojoa; y el Centro Regional de Educación Normal (CREN), ubicado en la ciudad de Navojoa. El estudio trabajó con registros académicos correspondientes a los primeros dos años, es decir, los primeros cuatro semestres de la cohorte seleccionada, así como datos del examen de ingreso (EXANI-II) y datos recabados por una medida de variables socioculturales.

La cohorte seleccionada contaba con un total de 282 estudiantes, de los cuales solo 268 sujetos cumplieron con los requisitos necesarios para participar en el estudio. Dichos requisitos fueron establecidos mediante criterios que permitieran elaborar los análisis correspondientes al origen social y al rendimiento académico. Los criterios para la selección de los sujetos fueron los siguientes:

- Contar con registro del promedio de bachillerato.

- Haber participado y contar con registro del examen de ingreso (EXANIIII).

- Estar inscrito en el semestre actual y tener reporte de las materias hasta el cuarto semestre de la LEP.

- Contar con los datos de evaluación sociocultural.

La recolección de la información de acuerdo con estos criterios se llevó a cabo gracias a la participación y al apoyo brindado por las instituciones participantes y al Instituto de Formación Docente del Estado de Sonora (IFODES).

\section{Instrumentos de obtención de información}

La medida de evaluación socioescolar de Vera y Aragón (2008) se ajustó a la población destino e integró un apartado sobre las condiciones familiares docentes. Esta medida, con variables manifiestas, evalúa el contexto al que pertenecen los estudiantes, el cual cuenta con 72 reactivos enfocados a datos generales, salud, trayectoria académica, aspectos socioeconómicos, condiciones y hábitos de estudio, evaluación de la institución, consumo cultural y conocimiento y uso de nueva tecnología.

La consistencia interna de la medida mostró valores alfa de Cronbach que van de 0,65 a 0,90, con cargas factoriales mayores a 0,30, valores de hasta 0,75 y un índice de adecuación KMO (prueba KaiserMeyer-Olkin), aceptable para llevar a cabo el análisis de componentes principales, donde la varianza después de una rotación varimax es siempre mayor a $50 \%$. Se integran los factores en tres rubros: condiciones y hábitos de estudio, evaluación de la institución y consumo cultural. De igual forma se anexó el rubro que permitiera conocer las relaciones familiares en primera línea (padres) y segunda línea (abuelos, tíos, primos y/o hermanos) de los estudiantes con la profesión docente; a este apartado se le llamó condiciones familiares docentes (Vera et al., 2016).

Origen SOCIOCULtURAL de LOS ESTUdiantes. Esta dimensión se realizó siguiendo la referencia metodológica del trabajo realizado por Casillas, Chain y Jácome. (2007), pero la diferencia radica en que en el presente estudio se consideraron cinco tipos distintos de capital para conformar el origen social de los estudiantes. Dichos capitales se construyeron a partir de diferentes indicadores contenidos en los distintos instrumentos de recolección de datos. Cada uno de estos indicadores fue reconfigurado estadísticamente de tal manera que permitiera determinar distintos niveles.

La construcción de índices permitirá categorizar el origen o nivel sociocultural de los alumnos en función del capital sociocultural $K S C=K S O+K E C$ $+K C U+K E s+K F a m$ (respectivamente, capital social, 
económico, cultural, escolar y familiar) y el capital social $K S O=P d o c+$ Pnormal + NivelEd, donde Pdoc corresponde a padres $y / u$ otros familiares dedicados a la docencia, Pnormal remite a padres y/o familiares con estudios en escuela normal y con nivel educativo: NivelEd. Se consideraron items correspondientes al bloque de condiciones familiares docentes de la medida socioescolar, en el que se integran indicadores como: padres y/u otros familiares dedicados a la docencia, padres y/o familiares con estudios en escuela normal y nivel educativo donde laboran padres $y / u$ otros familiares. Los familiares referidos en esta variable se dividieron en dos grupos: familiares en primera línea de consanguinidad (hermano/as) y familiares en segunda línea de consanguinidad (abuelo/ as, tío/as, primo/as). Con estos indicadores se logró caracterizar cuatro distintos niveles de capital social: nulo, bajo, medio y alto.

CAPITAL ECONÓMICO KEC $=E n+$ Ser + Tra . Suma los enseres disponibles en casa con los servicios con los que cuenta la casa habitación y el trabajo de la persona y utiliza los indicadores socioeconómicos de evaluación socioescolar, como: servicio telefónico, lavadora de ropa, horno de microondas y trabajo durante el bachillerato. La construcción de esta variable consideró como referente la metodología 10 × 6 utilizada por la Asociación Mexicana de Agencias de Investigación de Mercado (AMAI) para la definición de niveles socioeconómicos de la población mexicana (López, 2008), en cuatro distintos niveles: bajo, medio-bajo, medio y alto.

CAPITAL CULTURAL KCU $=$ EXIng + Cond + Hab. Construido mediante indicadores del examen de ingreso (EXANIIII) y del bloque de condiciones y hábitos de estudio del instrumento de evaluación sociocultural, estos indicadores miden la cantidad de libros, películas, horas de estudio fuera de la escuela normal y visitas a diversos estados de la república, así como la frecuencia con la que el estudiante realiza, asiste y lee cuestiones extracurriculares. Esta variable se caracterizó mediante tres niveles de capital cultural: bajo, medio y alto.
CAPITAL ESCOLAR KEs = Perma + Matrep + EXANI + Promsec + PromBach. Utiliza indicadores de permanencia/continuidad escolar y materias reprobadas, al igual que las calificaciones obtenidas en el examen de ingreso (EXANI) y el promedio obtenido en secundaria y bachillerato. Este capital se construyó mediante indicadores del bloque de datos generales del instrumento de evaluación sociocultural y las bases de datos proporcionadas por las instituciones que le fueron entregadas por CENEVAL. De igual manera, se caracterizó de acuerdo con tres niveles distintos de capital escolar: bajo, medio y alto.

CAPITAL FAmiliar $\mathrm{KFAM}=$ Pres + escPad + Escmad. Esta variable se sujetó a indicadores del bloque de datos general de la medida socioescolar relacionados con el prestigio de la ocupación y el nivel de escolaridad de los padres. Por otra parte, para determinar el prestigio de la ocupación registrada se utilizó el Sistema Nacional de Clasificación de Ocupaciones de 2011 (sInco) del Instituto Nacional de Estadística y Geografía (INEGI, 2011). Esta última variable también se caracterizó mediante cuatro niveles distintos de capital escolar: bajo, medio-bajo, medio y alto.

DIMENSIÓN RENDIMIENTO ACADÉmICO. Esta variable se construyó utilizando como indicadores los promedios generales de los estudiantes en cada uno de los primeros cuatro semestres, así como el promedio global, que incluye todas sus calificaciones. La obtención de estos promedios se hizo mediante los registros de las calificaciones obtenidas en cada uno de sus cursos o asignaturas contempladas en la malla curricular de primer a cuarto semestre de la LEP.

\section{Trabajo de campo}

En primera instancia, la recolección de información se llevó a cabo mediante la revisión de la base de datos proporcionada por el IFODEs en relación con el examen de los aspirantes a ingresar en la Generación 2011-15 de la LEP. Seguido a esto, se estableció contacto con las autoridades correspondientes de cada escuela normal participante para comuni- 
car el objetivo del proyecto y solicitar información referente a las listas de alumnos en activo de la generación 2011-15, así como sus calificaciones oficiales obtenidas hasta cuarto semestre de la LEP.

Primero se visitaron, por su ubicación, los planteles del CREN y la ENR, para establecer contacto personalmente con los encargados del puesto de secretario académico y solicitar a su vez los datos correspondientes al promedio de bachillerato de los participantes. Este mismo procedimiento se llevó a cabo en una fecha diferente con las autoridades del plantel de la ENES.

Para finalizar, se visitaron los planteles del CREN y la ENR para realizar la aplicación del instrumento Evaluación y Valores Socioculturales a los sujetos participantes, los cuales se encontraban en activo en el séptimo semestre de su licenciatura y a punto de iniciar su práctica docente. Debido al inicio de esta práctica docente, se tuvo que posponer dos meses la aplicación del instrumento a los tres grupos faltantes de la cohorte seleccionada correspondientes a la ENES.

Los instrumentos estaban foliados y correspondían a todos y cada uno de los alumnos de normales, y también se enfatizó en la confidencialidad de los datos y se firmaron los consentimientos informados; con el fin de evitar los valores perdidos, se revisó cada uno de los instrumentos aplicados para verificar que todas las preguntas quedaran contestadas.

Cada uno de los estudiantes llenó el formulario para cada una de las preguntas, entre 45 y 60 minutos; en ninguna ocasión se les entregó alguna recompensa física monetaria y se mantuvieron constantes todos los parámetros descriptivos-explicativos y de procedimiento en cada uno de los grupos de estudiantes normalistas. El trabajo de campo se realizó de acuerdo con los artículos 118 y 122 del Código Ético del Psicólogo (Sociedad Mexicana de Psicología, 2007), los cuales hacen referencia al consentimiento informado del estudiantado. Asimismo, la investigación también se basó en el artículo 136 de dicho Código ético, que hace referencia a la confidencialidad, ya que se infor- mó acerca de la utilización de los datos recopilados, así como del uso anónimo de los mismos.

\section{Análisis de datos}

Una vez construidas ambas dimensiones con sus respectivas variables, se comenzó a implementar los procedimientos estadísticos correspondientes con el paquete estadístico SPSS Versión 22. Los análisis y pruebas realizadas fueron: un análisis de conglomerados para K-medias y dos análisis de regresión por pasos.

\section{Resultados}

A continuación, se exponen los resultados obtenidos en el estudio. De inicio se presentan los referidos al origen social de los LEP, a partir de un análisis de conglomerados para K-medias. Seguido a esto, se dan los resultados de las dos pruebas de análisis de regresión, con los capitales predictores del rendimiento académico de los estudiantes de la LEP. El origen social se examinó mediante un análisis de clasificación por conglomerados o clúster de tipo no jerárquico, mediante el procedimiento de K-medias, introduciendo como variables las puntuaciones medias de los reactivos que corresponden a las escalas para estudiar los diferentes tipos de capitales. Se exploraron inicialmente los modelos de tres, cuatro y cinco componentes, donde el quíntuple pareció ser el más adecuado, pues no incluye números excesivamente reducidos de sujetos, como ocurrió con la opción de cuatro agrupamientos. Cabe señalar que para ningún grupo se encontraron elementos con más de tres desviaciones estándar con respecto a sus centroides. Esta prueba permitió determinar cinco perfiles diferentes de los estudiantes de la LEP, de acuerdo con sus características familiares, económicas, escolares, culturales y sus relaciones sociales con la carrera docente.

A continuación, se muestran en la Tabla 1 los estadísticos descriptivos y los puntajes generales alcanzados por los estudiantes para cada uno de los capitales que integran la dimensión de origen social 
(estos puntajes provienen de los valores asignados a cada indicador en la construcción de los capitales).

Tabla 1. Datos descriptivos de los capitales

\begin{tabular}{|c|c|c|c|c|c|c|}
\hline 苞 & 点产 & 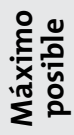 & 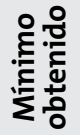 & 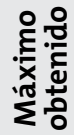 & $\underset{\sum}{\stackrel{\pi}{\overparen{D}}}$ & 㟔 \\
\hline Cultural & 1 & 3 & 1,10 & 2,90 & 1,95 & 0,34 \\
\hline Económico & 1 & 4 & 1,30 & 3,75 & 2,46 & 0,57 \\
\hline Escolar & 1 & 3 & 1,00 & 3,00 & 2,23 & 0,44 \\
\hline Familiar & 1 & 4 & 1,50 & 4,00 & 2,65 & 0,69 \\
\hline Social & 1 & 4 & 0,12 & 3,00 & 1,20 & 0,74 \\
\hline
\end{tabular}

Fuente: elaboración propia.

Por otro lado, en lo referente a la prueba de conglomerados de K-medias, las variables de capital escolar y capital cultural presentaron valores contrastantes con los presentados por el capital económico, familiar y social (Tabla 2). Sin embargo, todos presentaron una significancia de $p=0,000$, con excepción del capital escolar, que obtuvo $p=0,007$. A pesar de este contraste entre los valores de las variables, las distancias de cada uno de los centroides no mejoraron; solo se impidió la homogeneidad entre las distancias presentadas. Se debe mencionar que las distancias entre cada centroide fueron de un mínimo de 0,234 y un máximo de 1,428, manteniendo su rango dentro de las tres desviaciones estándar, tal como se puede observar en el Tabla 2.

Tabla 2. Centro y valores de F de conglomerados finales

\begin{tabular}{|c|c|c|c|c|c|c|}
\hline \multirow{2}{*}{ Capital } & \multicolumn{6}{|c|}{ Conglomerados } \\
\cline { 2 - 7 } & $\mathbf{1}$ & $\mathbf{2}$ & $\mathbf{3}$ & $\mathbf{4}$ & $\mathbf{5}$ & $\mathbf{F}$ \\
\hline Cultural & 2,05 & 1,87 & 2,14 & 1,87 & 1,85 & 8,94 \\
\hline Económico & 2,77 & 2,96 & 2,94 & 1,94 & 2,07 & 96,06 \\
\hline Escolar & 2,36 & 2,36 & 2,25 & 2,22 & 2,09 & 3,63 \\
\hline Familiar & 2,83 & 2,67 & 3,61 & 2,06 & 2,27 & 118,03 \\
\hline Social & 0,53 & 1,53 & 2,22 & 0,40 & 1,45 & 402,17 \\
\hline
\end{tabular}

Fuente: elaboración propia.
Con los resultados presentados por dicha prueba, se estableció que los capitales que marcaron una diferencia más clara en el origen social de los estudiantes de la LEP fueron el capital económico, familiar y social, en un orden de menor a mayor en relación con los valores de F. De igual manera, esta prueba permitió obtener cinco grupos distintos de origen social (Tabla 3).

- $\quad$ Clúster 1: estudiantes de origen social ilustrado, con padres que tienen estudios superiores, poca relación familiar con la profesión docente, con una trayectoria escolar buena y provienen de un sector económicamente medio.

- Clúster 2: sujetos con origen social popular; provienen de sectores económicamente altos, con padres con estudios de nivel superior y algunos familiares relacionados con la profesión docente, pero muestran un capital culturalmente bajo.

- $\quad$ Clúster 3: alumnos de origen social heredado; estos provienen de sectores altos de la sociedad, con excelentes recursos financieros y padres con estudios universitarios, además de tener bastantes relaciones familiares con la profesión docente y una trayectoria escolar previa aceptable.

- Clúster 4: estudiantes con origen social de héroes, lo que indica que, a pesar de no contar con recursos financieros óptimos y provenir de sectores socialmente bajos, poseen trayectorias escolares previas buenas, sumado a que el estudio de sus padres es de educación media superior. Son alumnos que trabajaron durante el bachillerato y trabajan durante sus estudios superiores. Su relación con la docencia es nula o con segundos familiares.

- Clúster 5: estudiantes con origen social marginado, que provienen de un sector medio-bajo de la sociedad. Poseen trayectorias escolares bajas y sus padres cuentan con estudios de 
nivel medio superior y ocupaciones de nivel medio. Son estudiantes que regularmente trabajan desde el bachillerato para mantener sus estudios, pero, a diferencia de los héroes, cuentan con relaciones familiares docentes.

Por otra parte, para conocer los tipos de capital que afectan la variable de rendimiento académico, se realizaron dos análisis de regresión por pasos; para ello, se utilizaron como variables dependientes el promedio general alcanzado durante el primer semestre (en el primer análisis) y el promedio general del cuarto semestre (en el segundo análisis). Como variables predictivas, se utilizaron en ambas regresiones los diversos tipos de capitales construidos: social, cultural, familiar, escolar y económico.

Como requisito estadístico se realizó una prueba de correlaciones bivariadas de Pearson para determinar el criterio de colinealidad, indispensable para las relaciones de los análisis de regresión (Tabla 4). De igual forma, para cada prueba de regresión por pasos se realizaron las pruebas de tamaño del efecto y potencia estadística correspondientes.

En lo referente a las pruebas de regresión, la primera presentó un valor estadístico de DurbinWatson de 1,61, lo cual indica independencia en los residuos (Tabla 5). El modelo contrastó la hipótesis a través de la prueba ANOva, donde el valor de R fue significativamente distinto a cero. La raíz cuadrada de la media cuadrática corregida es igual a 0,266, puntaje referente a la variabilidad que logra predecir las variables independientes que no son explicadas por la recta de regresión de los residuos. Estos resultados indicaron que entre las variables que predijeron el resultado del promedio general del primer semestre está, solamente, el capital escolar, el cual hace referencia al desempeño escolar del alumno en niveles educativos anteriores.

Tabla 3. Nivel de los capitales y número de casos presentados en los conglomerados finales

\begin{tabular}{|c|c|c|c|c|c|c|}
\hline Clúster & $\begin{array}{c}\text { No. de } \\
\text { casos }\end{array}$ & $\begin{array}{c}\text { Nivel de capital } \\
\text { cultural }\end{array}$ & $\begin{array}{c}\text { Nivel de capital } \\
\text { económico }\end{array}$ & $\begin{array}{c}\text { Nivel de capital } \\
\text { escolar }\end{array}$ & $\begin{array}{c}\text { Nivel de capital } \\
\text { familiar }\end{array}$ & $\begin{array}{c}\text { Nivel de capital } \\
\text { social }\end{array}$ \\
\hline 1 & 49 & Medio & Medio & Medio & Medio & Majo \\
\hline 2 & 36 & Bajo & Alto & Medio & Medio & Alto \\
\hline 3 & 55 & Medio & Alto & Medio & Medio-bajo & Nulo \\
\hline 4 & 63 & Bajo & Bajo & Medio & Medio-bajo & Medio \\
\hline 5 & 65 & Bajo & Medio-bajo & Bajo & & \\
\hline
\end{tabular}

Nota: el número total de casos es de 268 , con o valores perdidos.

Fuente: elaboración propia.

Tabla 4. Correlaciones entre variables dependientes y capitales

\begin{tabular}{|c|c|c|c|c|c|c|c|}
\hline $\begin{array}{c}\text { Variables } \\
\text { dependientes }\end{array}$ & Media & DE & $\begin{array}{c}\text { Capital } \\
\text { cultural }\end{array}$ & $\begin{array}{c}\text { Capital } \\
\text { económico }\end{array}$ & $\begin{array}{c}\text { Capital } \\
\text { escolar }\end{array}$ & $\begin{array}{c}\text { Capital } \\
\text { familiar }\end{array}$ & $\begin{array}{c}\text { Capital } \\
\text { social }\end{array}$ \\
\hline $\begin{array}{c}\text { Promedio de } 1^{\text {er }} \\
\text { semestre }\end{array}$ & 88,49 & 6,57 & 0,075 & 0,095 & $0,518^{* *}$ & 0,086 & $-0,058$ \\
\hline $\begin{array}{c}\text { Promedio de } 4^{\text {to }} \\
\text { semestre }\end{array}$ & 90,66 & 5,82 & 0,106 & $0,323^{* *}$ & $0, .508^{* *}$ & $0,243^{* *}$ & 0,006 \\
\hline
\end{tabular}

${ }^{*} p<0,05 ;{ }^{* *} p<0,01$.

Fuente: elaboración propia. 
En el caso de la segunda prueba de regresión, el valor estadístico de Durbin-Watson fue de 1,45, que indica independencia en los residuos. El modelo, de igual manera, contrastó la hipótesis a través de la prueba de ANOVA, donde el valor de $\mathrm{R}$ fue significativamente distinto a cero. La raíz cuadrada de la media cuadrática corregida resultó igual a o,460. Las variables que predijeron el resultado del promedio general de cuarto semestre en el segundo análisis de regresión fueron el capital escolar y el capital económico (Tabla 5).

\section{Discusión y conclusiones}

De los resultados obtenidos, se resalta la importancia de la relación familiar del estudiante con la profesión docente (capital social), la cual tuvo el mayor peso de diferenciación entre los diferentes perfiles de origen social, debido a que la población estudiada presentó un $63,1 \%$ de relación con la profesión docente, es decir, que el estudiante tenía por lo menos un familiar docente, ya fuera en primera o segunda línea de consanguinidad, lo cual nos lleva a considerar que aún existe algún criterio de prestigio asociado a la tradición familiar. De acuerdo con Bourdieu y Passeron (2009), los estudiantes con origen social hereditario son los que tienen mayores probabilidades de éxito para ingresar a la carrera docente, y aunque el ingreso a la profesión docente actualmente se da por concurso de oposición, que brinda igualdad de oportunidades para los aspirantes, los estudiantes de origen más favorecidos presentan una ventaja social para un rápido ingreso al campo laboral, por su familiarización con los procedimientos y prácticas políticas inmersas en el sistema educativo.

De igual manera, las características económicas del grupo siguen marcando diferencia como una variable importante, al igual que la profesión y escolaridad de los padres (capital familiar), lo que corrobora los resultados encontrados por Yáñez et al. (2013), al establecer que 60\% de los estudiantes normalistas que logran ingresar a las escuelas normales en Sonora presentan como antecedente familiar y económico el provenir de familias cuyo ingreso per cápita se encuentra por debajo de la línea de bienestar mínimo establecido por coneval (INEE, 2015). Por lo anterior, el capital económico resulta una variable importante en el cuarto semestre, lo que indica que aquellos más afortunados, denominados herederos, mejoran sus promedios semestrales.

Así mismo, las similitudes obtenidas entre las características de los estudiantes y el factor geográ-

Tabla 5. Análisis de regresiones por pasos para las variables predictivas del promedio general de primer semestre y general del cuarto semestre

\begin{tabular}{|c|c|c|c|c|c|}
\hline Paso y variable predictora & B & SE B & B & $\mathbf{R}^{2}$ & $\mathbf{R}^{2}$ \\
\hline \multicolumn{7}{|c|}{ Modelo 1 } \\
\hline \multicolumn{7}{|c|}{ Paso 1: } \\
\hline Capital escolar & 7,66 & 0,776 & 0,518 & $0,266^{*}$ & \\
\hline \multicolumn{7}{|c|}{ Modelo 2 } \\
\hline \multicolumn{7}{|c|}{ Paso 1: } \\
\hline Capital escolar & 8,73 & 0,634 & 0,621 & $0,411^{*}$ & $0,14^{*}$ \\
\hline \multicolumn{7}{|c|}{ Paso 2: } \\
\hline Capital económico & 2,49 & 0,488 & 0,231 & $0,460^{*}$ & $0,049^{*}$ \\
\hline
\end{tabular}

${ }^{*} p<0,05$.

Fuente: elaboración propia. 
fico o región donde se encuentra inmersa la escuela normal se relacionan fuertemente con el origen social de los estudiantes, factor que Bourdieu y Passeron (2009) consideran importante como oportunidad para incrementar los capitales. La relación entre origen social y asignación de ayudas estudiantiles encontrada en el estudio de Quintela (2013) es refutada por los resultados obtenidos con relación al origen social heroico y marginal, los cuales presentan niveles económicos medios-bajos y bajos, en su mayoría pertenecientes a la Escuela Normal Rural El Quinto, ubicada en una comunidad rural del estado de Sonora. Estos estudiantes contaban con recursos financieros y más del 50\% tuvieron becas durante la preparatoria. El sustento por medio de becas de apoyo del gobierno federal es importante para asegurar el ingreso y la permanencia en estas escuelasinternados de formación del magisterio. Con ello se muestra la importancia de las escuelas normales rurales como opción de educación superior para los sectores del campo, las cuales han sido, en su perfil histórico, uno de los pocos medios educativos para la preparación profesional docente de los habitantes de dicho sector (Civera y Antón, 2018). Por su parte, los orígenes sociales de herederos, populares e ilustrados se encuentran en instituciones ubicadas en zonas urbanas (ENES y CREN) y sus recursos financieros, así como sus bajos índices de becas por necesidad económica en el bachillerato, muestran relación con el estatus social.

El estudio mostró un mayor porcentaje de estudiantes de género femenino en la Licenciatura en Educación Primaria en Sonora, cantidad semejante a los porcentajes nacionales de los docentes activos en educación primaria, donde las mujeres muestran un $67,3 \%$ en los porcentajes de ingreso a las escuelas normales, donde la mayor parte de los estudiantes son mujeres (INEE, 2015). Este fenómeno también se observa en la conformación de cuerpos académicos dentro de las normales en México, donde más de la mitad de los integrantes son mujeres (Siqueiros y Vera, 2020). Por último, con relación a su situación económica, entre el 38\% y 46\% recibe algún apoyo económico adicional para realizar sus estudios y cerca del 30\% solo cuenta con una beca de estudio.

Por tal motivo, se concluye que el trabajo realizado solo aborda parte de los retos contemplados para la investigación relacionada con el estudio de la formación inicial y continua de los profesores de educación básica. Este conocimiento puede ser de interés para mejorar y fortalecer tanto los programas institucionales de asesorías implementados en las escuelas normales como las reformas curriculares destinadas a estos estudiantes. En primera instancia, pueden considerarse para la mejora de los criterios del perfil de ingreso incluir, además del promedio, algunos aspectos del capital cultural, particularmente el vocacional e identitario que se trasmiten dentro de la familia. Por otro lado, se debe incluir la opinión de los alumnos en el diseño de políticas de formación inicial, ya que se realizan sin siquiera conocer las características de los actores a quienes van dirigidas ni quiénes serán encargados de ejecutarlas, lo cual tiene relación con el desconocimiento de la planta docente de las escuelas normales.

Trabajos como el informe presentado por el INEE en 2015 acortan la brecha en el conocimiento de estadísticas sobre la formación de los profesores, pero no se está cerca de conocer su realidad. Uno de los problemas observados es precisamente el considerar a los estudiantes normalistas como entes homogéneos que solo se preparan para ser docentes y laborar en educación básica. Las escuelas normales van más allá de la formación de profesores de primaria y preescolar, debido a que dentro del sistema también se encuentran docentes-investigadores, directivos y supervisores, quienes se formaron y laboraron inicialmente como profesores (Del Cid et al., 2021), además que se trata de una población muy heterogénea en sus capitales culturales (Arnaut, 2004).

El estudio mostró cómo el origen social de los estudiantes normalistas de una cohorte específica se encuentra definido en sus características generales 
por el contexto en el cual está inmersa la escuela normal a la que asisten, y determinó que los estudiantes que se encuentran ubicados en las zonas urbanas poseen mayor capital económico y social. Es importante mencionar que el capital social es el principal factor para determinar los diferentes perfiles de origen social encontrados, debido a las relaciones de las redes familiares con la profesión docente, tal como muestran los estudiantes de la LEP analizados. Asimismo, el capital social permite analizar que estas relaciones familiares se extienden más allá de los padres, así que, si ensanchamos la relación a familiares de segunda línea (hermanos, primos, tíos y abuelos), encontraremos que aún se mantiene una tradición familiar docente. Por lo anterior, el ethos es un factor importante para la permanencia de los individuos en el sistema educativo de las normales (Moreno, 2014).

Los orígenes sociales encontrados demuestran que la desigualdad social sigue siendo un factor que interviene en el logro educativo de los estudiantes, hecho que constatan indicadores como el factor económico, el cual se mantiene como propicio para el rendimiento académico de los estudiantes. Así lo demostraron las pruebas de regresión, donde el capital escolar probó ser el factor con mayor predicción durante el primer semestre, dato que concuerda con lo obtenido por Tapasco et al. (2019); en tal sentido, conforme se da el avance en los semestres, el capital económico toma valor de predicción como factor de rendimiento académico en el promedio final de los semestres y en el resultado del examen intermedio de conocimiento (Araiza, Ibarra y Audelo, 2018; Yáñez, Vera y Mungarro, 2013).

En cuanto a los límites de este estudio, que deben considerarse en próximas investigaciones, en primer lugar, aun cuando la muestra es representativa de los estudiantes de la licenciatura en educación primaria de Sonora, se requiere extenderla a los do- centes de preescolar y secundaria, y, en segundo lugar, hay que realizar pruebas criteriales contando con los datos de logro académico en el cuarto semestre.

Además, se sugiere la realización de investigaciones de corte mixto o cualitativo que permitan analizar las variables de identidad y vocación, ya que el vínculo histórico familiar con la profesión parece constituir un aspecto fundamental, no solo para los agrupamientos, sino también para las regresiones. Parecería que la norma social subjetiva (Ajzen y Fishbein, 1997), así como la percepción de la persona sobre las presiones sociales que le son impuestas, lleva a identificarse con el magisterio y a considerar las valoraciones sociales que se le otorgan a la profesión, lo cual redunda en la visión del joven acerca de su comportamiento y motivación general para actuar conforme a las normas. Durante las etapas tempranas de educación secundaria y media superior, estas normas impuestas sobre las ventajas personales, sociales y familiares de convertirse en profesor son más favorables cuando se acompañan de un modelamiento de consumo cultural alto, donde la literatura, el cine y las artes son un componente del proceso formativo.

En la prospectiva de investigación, deberemos considerar que los continuos cambios en las políticas educativas del Estado y las transiciones en la cantidad de proyectos escolares hacen indispensable pensar en la identidad con la profesión docente, como factor relevante en la formación y el ejercicio docente en educación básica (Sayago, Chacón y Rojas, 2008). Es importante mencionar que se trata de una profesión femenina que ha venido transitando hacia la equidad y, sin embargo, se requiere investigación cualitativa sobre las estrategias de afiliación que la familia utiliza para convencer e identificar a la adolescente con el magisterio y compararla con los procesos subyacentes en jóvenes (González, 2009). 


\section{Referencias}

Ajzen, I. y Fishbein, M. (1977). Attitude-behavior relations: A theoretical analysis and review of empirical research. Psychological Bulletin, 84(5), 888-918. DOI: https://doi.org/10.1037/0033-2909.84.5.888

Arnaut, A. (2004). El sistema de formación de maestros en México. Continuidad, reforma y cambio. Cuadernos de Discusión 17. Secretaría de Educación Pública de México. Recuperado de: http://www.formaciondocente. com.mx/BibliotecaDigital/12_FormacionContinua/03\%20Cuadernos\%2Ode\%20Discusion\%20el\%2oSistema\%20de\%2oFormacion\%20en\%20Mexico.pdf

Araiza, M., Ibarra, E. y Audelo, C. (2018). Desigualdades socioeducativas y rendimiento académico de los estudiantes de la Universidad Politécnica de Sinaloa. Praxis Investigativa ReDIE, 10(19), 141-153. Recuperado de: http://oaji.net/articles/2019/7222-1568659920.pdf

Araiza, M. y Audelo, C. (2017). El origen social de los estudiantes universitarios y su trayectoria escolar universitaria. [Ponencia] XIV Congreso Nacional de Investigación Educativa, San Luis Potosí, México, noviembre. Recuperado de: http://www.comie.org.mx/congreso/memoriaelectronica/v14/doc/1331.pdf

Arango, L., Quintero, O. y Mendoza, I. (2004). Género y origen social en el acceso a la universidad nacional: trayectoria de estudiantes de sociología y de ingeniería de sistemas. Revista Colombiana de Sociología, 22, 87-110. DOI: http://dx.doi.org/10.15446/rcs

Barahona U., P. (2014). Factores determinantes del rendimiento académico de los estudiantes de la Universidad de Atacama. Estudios Pedagógicos, 40(1), 25-39. DOI: http://dx.doi.org/10.4067/S0718-07052014000100002

Bernardi, F. y Cebolla, H. (2014). Clase social de origen y rendimiento escolar como predictores de las trayectorias educativas. Revista Española de Investigaciones Sociológicas, 146, 3-22. DOI: https://doi.org/10.5477/cis/reis.146.3

Bourdieu, P. (1979). Los tres estados del capital cultural. Sociológica, 2(5). Recuperado de: http://www.sociologicamexico.azc.uam.mx/index.php/Sociologica/article/view/1043

Bourdieu, P. y Passeron, J. (2009). Los herederos, los estudiantes y la cultura. Siglo XXI.

Bracho, T. (1990). Capital cultural: impacto en el rezago educativo. Revista Latinoamericana de Estudios Educativos, 20(2), 13-46. Recuperado de: http://www.cee.edu.mx/revista/r1981_1990/r_texto/t_1990_2_o2.pdf

Cárcamo, H.y Rodríguez, C. (2015). Rol parental educativo: aproximación a las percepciones que poseen los futuros profesores. Educación y Educadores, 18(3), 456-470. DOI: http://dx.doi.org/10.5294/edu.2015.18.3.5

Casillas, M., Chain, R. y Jácome, N. (2007). Origen social de los estudiantes y trayectorias estudiantiles en la Universidad Veracruzana. Revista de Educación Superior, 36(2), 7-29. Recuperado de: http://publicaciones. anuies.mx/pdfs/revista/Revista142_S1A1ES.pdf

Chaparro, A., González, C. y Caso, J. (2016). Familia y rendimiento académico: configuración de perfiles estudiantiles en secundaria. Revista Electrónica de Investigación Educativa, 18(1). Recuperado de: https://redie.uabc. $\mathrm{mx} /$ redie/article/download/774/1337 
Civera, A. y Antón, R. (2018). Desde la historia de la educación: educación y mundo. Historia y Memoria de la Educación, 7, 9-45. DOI: https://doi.org/10.5944/hme.7.2018

Da Cuña, l., Gutiérrez, M., Barón, F. y Labajos, M. (2014). Influencia del nivel educativo de los padres en el rendimiento académico, las estrategias de aprendizaje y los estilos de aprendizaje, desde la perspectiva de género. Revista de Estilos de Aprendizaje, 7(13), 64-84. Recuperado de: https://dialnet.unirioja.es/servlet/ articulo?codigo $=4728984$

Del Cid, C., Estévez, E., González, E.y Vera, J. A. (2021). Efectos de programas de política pública sobre las actividades e identidad de la profesión académica en las escuelas Normales de México. Perfiles Educativos, 43(171), 63-81. DOI: https://doi.org/10.22201/iisue.24486167e.2021.171.59671

DGESPE - Dirección General de Educación Superior para Profesionales de la Educación (2013). Programa de Fortalecimiento Académico de los Estudiantes de las Escuelas Normales. Secretaría de Educación Pública de México. Recuperado de: https://www.dgespe.sep.gob.mx/web_old/pemde

Enciso, M. (2013). El origen social de los graduados y la equidad en el acceso a la universidad. Revista de la Educación Superior, 42(165), 11-29. Recuperado de: http://www.scielo.org.mx/scielo.php?script=sci_ arttext\&pid=S0185-27602013000100002

Fonseca, G. (2018). Trayectorias de permanencia y abandono de estudios universitarios: una aproximación desde el currículum y otras variables predictoras. Educación y Educadores, 21(2), 239-256. DOI: http://dx.doi. org/10.5294/edu.2018.21.2.4

Flores, M., Stadhagen, H. y Reyes, N. (2014). Una mirada al capital cultural de los estudiantes de la Universidad Autónoma del Estado de México. Contribuciones desde Coactepec, 14(27), 117-140. Recuperado de: http:// www.redalyc.org/html/281/28133880007/

Garbanzo, G. (2013). Factores asociados al rendimiento académico en estudiantes universitarios desde el nivel socioeconómico: un estudio en la Universidad de Costa Rica. Revista Electrónica Educare, 17(3), 57-87. Recuperado de: http://www.redalyc.org/pdf/1941/194128798005.pdf

González, J. R. (2009). De cómo y por qué las maestras llegaron a ser mayoría en las escuelas primarias de México, Distrito Federal (finales del siglo XIX y principios del XX): un estudio de género. Revista Mexicana de Investigación Educativa, 14(42), 747-85. Recuperado de: http://www.scielo.org.mx/pdf/rmie/v14n42/ v14n42a5.pdf

González, E. (2013). Estudio sobre factores contexto en estudiantes universitarios para conocer por qué unos tienen éxito mientras otros fracasan. Revista Intercontinental de Psicología y Educación, 15(2), 135-154. Recuperado de: http://www.redalyc.org/articulo.oa?id=80228344008

Ibarrola, M. (2014). Repensando las relaciones entre la educación y el trabajo: una reflexión basada en investigaciones realizadas en México. Cuadernos CEDES, 34(94), 367-383. DOI: http://dx.doi.org/10.159o/ So101-32622014000300006 
Instituto Nacional de Estadística y Geografía (2011). Sistema Nacional de Clasificación de Ocupaciones. Recuperado de: http://www3.inegi.org.mx/sistemas/clasificaciones/sinco/sinco.aspx

INEE - Instituto Nacional para la Evaluación de la Educación (2015). Los docentes en México, Informe 2015. INEE. Recuperado de: http://publicaciones.inee.edu.mx/buscadorPub/P1///240/P1/240.pdf

INEE - Instituto Nacional para la Evaluación de la Educación (2017). Panorama educativo de México 2016. Indicadores del Sistema Educativo Nacional. Educación básica y media superior. INEE. Recuperado de: http:// publicaciones.inee.edu.mx/buscadorPub/P1/B/115/P1B115.pdf

López, H. (2008). Nuevo Índice de Nivel Socioeconómico AMAl. [Ponencia] Congreso AMAl 2008, Ciudad de México, septiembre. Recuperado de: http://www.amai.org/congreso/2008/memorias/ponencias/lopezromo.pdf

Marqués, I. y Gil, C. (2015). Origen social y sobreeducación en los universitarios españoles: ¿es meritocrático el acceso a la clase de servicio? Revista Española de Investigaciones Sociológicas, 150, 89-112. DOI: https://doi. org/10.5477/cis/reis.150.89

Medrano, V., Ángeles, E. y Morales, M. (2017). La educación normal en México. Elementos para su análisis. INEE.

Moreno, E. (2014). La lectura académica y la teoría sociológica de Pierre Bourdieu. Enunciación, 19(2), 292-304. Recuperado de: https://revistas.udistrital.edu.co/ojs/index.php/enunc/article/view/7533

Quintela, G. (2013). Desigualdades sociales y educación superior: el proceso de toma de decisiones de estudiantes desde un enfoque sociológico. En M. Irigioin, R. del Valle y M. Ayala (eds.), Acceso y permanencia en la educación superior: Sin apoyo no hay oportunidad (pp. 53-96). AEovalis.

Sayago, Z., Chacón, M. y Rojas, M. (2008). Construcción de la identidad profesional docente en estudiantes universitarios. EDUCERE, 12(42), 51-561. Recuperado de: http://ve.scielo.org/pdf/edu/v12n42/art16.pdf

Sandoval, E. (2013). El trabajo docente. Entre cambios y continuidades. En C. Saucedo, C. Guzmán, E. Sandoval y J. Galaz (coords.), Estudiantes, maestros y académicos en la investigación educativa: Tendencias, aportes y debates 2002-2012 (pp. 289-318). ANUIES, COMIE.

Sandoval, E. y Villegas, N. (2013). Ser maestro: prácticas docentes en contextos escolares. En C. Saucedo, C. Guzmán, E. Sandoval y J. Galaz (coords.), Estudiantes, maestros y académicos en la investigación educativa: Tendencias, aportes y debates 2002-2012 (pp. 319-341). ANUIES, COMIE.

Santiago, P., McGregor, I., Nusche, D., Ravela, P. y Toledo, D. (2012). Revisiones de la OCDE sobre la evaluación en educación. OCDE, SEP, INEE. Recuperado de: http://publicaciones.inee.edu.mx/buscadorPub/P1/C/231/P1C231.pdf

Simón, H., Casado, J., Castejón, J. y Driha, O. (2017). ¿Existe alguna relación entre el tipo de centro de educación secundaria y el rendimiento académico universitario? En R. Roig-Vila (ed.), Investigación en docencia universitaria: diseñando el futuro a partir de la innovación educativa (pp. 814-824). Octaedro. Recuperado de: http://rua.ua.es/dspace/handle/10045/71081 
ISSN 0123-1294 | e-ISSN 2027-5358 | Educ.Educ. Vol. 24. No.1 | Febrero-abril de 2021 | pp. 53-70

Universidad de La Sabana | Facultad de Educación

Siqueiros, M. y Vera, J. A. (2020). Caracterización de cuerpos académicos de escuelas normales. CPU-e. Revista de Investigación Educativa, 31, 71-96. Recuperado de: https://cpue.uv.mx/index.php/cpue/article/view/2702

Sociedad Mexicana de Psicología (2007). Código ético del psicólogo. Trillas.

Tapasco, O., Ruiz, F., Osorio, D. y Ramírez, D. (2019). Deserción estudiantil: incidencia de factores institucionales relacionados con los procesos de admisión. Educación y Educadores, 22(1), 81-100. DOI: http://dx.doi. org/10.5294/edu.2019.22.1.5

Vera, J. A. y Aragón, R. (2008). Diseño y piloteo de medidas de aprendizaje en educación media superior en el estado de Sonora. Revista Ra Ximhai, 4(2), 67-77. Recuperado de: http://www.redalyc.org/pdf/461/46140204.pdf

Vera, J. A., Rodríguez, C. K., Huesca, L. y Laborín, F. (2016). Variables de contexto asociadas al desempeño en educación media superior para el estado de Sonora. CPU-e. Revista de Investigación Educativa, 22, 98-119. Recuperado de: http://www.redalyc.org/pdf/2831/2831435500o6.pdf

Yáñez, A., Vera, J. y Mungarro, J. (2013). El proceso de admisión de las escuelas normales y los antecedentes socioeconómicos como predictores del rendimiento académico. Revista Intercontinental de Psicología y Educación, 16(2), 111-129. Recuperado de: http://www.redalyc.org/html/802/80231541007/index.html 